\title{
Research on the Approximation Properties of Bandelet Transform Yong Huang ${ }^{1,}$ Yanchun Zhao ${ }^{1,}$ Zefu Zhao ${ }^{1}$ \\ ${ }^{1}$ College of Mathematics and Statistics, Zhaotong University, Zhaotong, Yunnan, 657000 \\ a email
}

\author{
Keywords: Approximation Property, Bandelet Transform, Mathematics
}

\begin{abstract}
Bandelet transform has undergone two generations of transformation theory development, its main application areas are image sparse expression and image compression, which penec for the first bandelet transform has made an important contribution, it not only involved in the first generation bandelet transform theory, The bandelet transform to improve and develop, greatly enhance its practical application. In this paper describes the bandelet transform, bandelet transform in-band approximation characteristics and global approximation characteristics of the expansion analysis.
\end{abstract}

\section{Bandelet Transform and Its Principle}

First, the bandelet transform defines a geometric vector line, which is used to represent the local regular direction of the image. The subdivision method is used to subdivide the support interval S of the image, $\mathrm{S}=\mathrm{U} \Omega$; Only a single contour line is included in each of the small subdivision intervals Q. However, the gray-scale variation in the area $\mathrm{Q}$ which does not contain any contour lines is uniformly regular, and for these local areas, It is necessary to define any geometric vector line. The geometric normal direction of the local area Q containing a contour line is the tangent direction of the contour line, and the sub-area containing the contour line is marked as the horizontal and vertical area. (X1, X2) on the local region $\Omega$ can be calculated according to the local geometric normal direction. Then, bandeletization of the bandelet block can be performed by using the interval wavelet along the vector flow. And the bandelet basis of the image itself can be fully utilized by this processing, and the set of bandelet bases on the whole subdivision region form a set of standard orthonormal bases on L (S).

In the geometric direction, assuming that the image equation is regular, the change of the image along the direction parallel to the edge line is regular, and the direction perpendicular to the edge line changes violently for the geometric regular image \$ Geometric (X1, X2) defined in the support region of the image, which is used to describe the direction of the regular change in each point. The direction parallel to the edge line can be characterized by a geometric flow.

The primary purpose of the bandelet transform is to make full use of the regularity of the edge along the geometrical flow. The geometric flow is generally parallel to the tangent direction of the edge line. For geometric regular images, the geometric flow is generally parallel.

The bandelet base is the core of the bandelet transform. To construct the bandelet base, the quadtree structure is firstly established to find the geometric direction of the image.

$(X, y)$, then the original image is $f(x, y)$, then $f(x, y)$ is the original image, then the original image is decomposed into two-dimensional biorthogonal discrete wavelet transform, and then the transformed multi-scale decomposition coefficient map is further processed. The two-dimensional wavelet transform is:

$$
\begin{aligned}
& <f, \psi_{j, m}>f^{*} \psi_{j, m}\left(m_{1} 2^{j}, m_{2} 2^{j}\right)\left(j \in Z, m_{1} m_{2}\right) \in Z^{2}, s \in\{H, V, D\} \\
& \left\{\begin{array}{l}
\psi_{j, m}=\varphi_{j, m}\left(x_{1}\right) \varphi_{j, m}\left(x_{2}\right) \\
\psi_{j, m}^{v}=\psi_{j, m}\left(x_{1}\right) \varphi_{j, m}\left(x_{2}\right) \\
\psi_{j, m}^{D}=\psi_{j, m}\left(x_{1}\right) \psi_{j, m}\left(x_{2}\right) \\
\psi_{j, m}^{H}=\varphi_{j, m}\left(x_{1}\right) \psi_{j, m}\left(x_{2}\right)
\end{array}\right.
\end{aligned}
$$


Which $\varphi_{j, m}(x)$ represent coarse approximation $\psi_{j, m}^{H}(x), \psi_{j, m}^{v}(x), \psi_{j, m}^{D}(x)$ are the high frequency information of horizontal, vertical and diagonal sub-bands, respectively, while scaling and translation $\psi(t)$ of scale function $\varphi(t)$ and wavelet function are respectively:

$$
\begin{aligned}
& \psi_{j, m}(t)=1^{-i / 2} \psi\left(2^{-j} t-k\right) \\
& \varphi_{j, m}(t)=1^{-i / 2} \varphi\left(2^{-j} t-k\right)
\end{aligned}
$$

For the multi-scale images obtained in the transformation $f_{j}^{*}$, except for the low-frequency part, the transforms are sparse in three directions at each scale, and the transform coefficients in each direction are further processed by binary quadtree division. The subband is divided into four small subbands and then divided into four smaller subbands for each subband in the next layer division until the minimum size is reached and the decomposition is stopped. , Is the desired quad-tree structure.

Bandeletization process is to store the two-dimensional wavelet coefficients in the bandelet block, resample them along the direction of optimal geometric flow, generate one-dimensional data, and then transform the one-dimensional data by one-dimensional wavelet transform. The direction of the optimal geometric flow is one-dimensional wavelet transform to find the direction of discontinuity, and then the wavelet transform is carried out along these directions. In addition, two-dimensional information can be projected into one-dimensional information along the optimal geometric direction Method to transform the two-dimensional line singularities into point singularities.

According to experience, set the quantization threshold $\mathrm{T}$; 3) using the biorthogonal wavelet transform method to carry on the wavelet transform processing to the input image; 4) In view of the wavelet (Wavelet Transform), the wavelet transform is used in the wavelet transform; And then the quadratic tree is divided into four sub-bands, and then the best geometric flow direction in each segment is calculated; 5) bandelet processing is performed for each bandelet block, that is bandelet subband, and Storing the respective band -let coefficients; 6) arranging the obtained bandelet coefficients in the form of corresponding matrix according to the basic principle and method of bandelet transform; 7) obtaining the quadtree structure, the best geometric flow direction and the bandlet coefficients are processing The end result.

\section{Bandelet Transform in Band Approximation}

Suppose that $\mathrm{F}(\mathrm{x}, \mathrm{y})$ denotes a fuzzy kernel, and performs scaled transformation on the fuzzy kernel. The mathematical form is:

$$
J(x, y)=r^{-2} J_{1}\left(\frac{x}{r}, \frac{y}{r}\right)
$$

$\mathrm{J}$ is supported on the set $[-1,1]$, then there exists:

$$
\left.\begin{array}{l}
\left\|J_{1}\right\|_{1}=\left\|J_{1}\right\|_{1} \\
\left\|J_{1}\right\|_{D^{\beta}}=r^{-(2+\beta)} \Leftrightarrow\left\|J_{1}\right\|_{D^{\beta}}=1 .
\end{array}\right\}
$$

The fuzzy kernel $\mathrm{J}$ ( $\mathrm{x}, \mathrm{y}$ ) can impose processing on the edge of the image. The range of this process appears as a band, denoted by $\mathrm{A}$, and the distance $\mathrm{E}$ from the point $\mathrm{A}$ in the region satisfies the following relation:

$$
A_{r}=\{(x, y) \mid e((x, y), E)<r\}
$$

The approximation properties of bandelet transform in region A are analyzed.

The principle of the bandelet transform begins with the geometry flow, and the construction of the corresponding basis functions is also homologous. Therefore, to analyze the in-band approximation properties of the bandelet transform, we need to start from the approximation of the geometric flow. "For the approximation effect of the geometric flow, To make it optimal, the number of control parameters must be reduced as much as possible. 
Assuming that A can obtain the projection on the x-axis, and the projection interval is in [a, b], we can calculate the interval length: $d=$ ba. For the approximation of geometric flow, we can use the orthogonality function of scale $2 \mathrm{k}$; For example, in this set of orthonormal functions $\left\{f_{k, m}(t) \mid d<m<d_{1} 2^{-k}\right\}$, the set of functions $f\left(2^{-k} t-m\right)$ contained in [a, b] can be described by

$$
h(t)=\sum_{m=1}^{d_{1} 2^{-k}} \beta_{m} f_{k, m}(t)
$$

If $\left\{f_{k, m}(t) \mid d<m<d_{1} 2^{-k}\right\}$ the space $\mathrm{W}$ contains a polynomial of order $\mathrm{q}$, then $\mathrm{f}(\mathrm{t})$ is a differentiable function that closely supports q order. "Based on this, $\mathrm{h}(\mathrm{t})$ in the scale size $2^{\mathrm{k}}$ and (b-a) $2^{-\mathrm{k}}\{\beta\}=0$ and $\left|h^{\prime}(x)\right|<2$ there is a constant $\mathrm{C}$, this constant is only associated with the scaling function $\mathrm{f}(\mathrm{t})$, and this constant makes the inequality $\left|\beta_{m}\right|<C(b-a)$ valid.

Assuming that the exact feature of the edge is denoted by $\mathrm{E}(\mathrm{X})$, the estimate of the edge feature is denoted by $\mathrm{E}(\mathrm{x})$ "since the existence of the fuzzy kernel $\mathrm{J}(\mathrm{x}, \mathrm{y})$ is unknown and $\mathrm{J}(\mathrm{x}, \mathrm{y})$, Then $\mathrm{E}$ $(\mathrm{X})$ in the approximation of the true value of the existence of a certain error, which $\|\tilde{E}-E\|<C_{E} r$ accords with the parameters Ce calculated by the computational precision constraints.

The flow integral is calculated from the projection of $\mathrm{E}(\mathrm{x})$ over the space Wk, that is,

$$
\begin{aligned}
& h(x)=Q_{w}, \tilde{E}(x)=\sum_{m=1}^{d_{1} 2^{-k}} \beta_{m} f_{k, m}(x) \\
& \beta_{m}=\left\langle\tilde{E}(x), f_{k, m}\right\rangle\left\|f_{k, m}\right\|^{-2}
\end{aligned}
$$

The parameter Ce and the scale $2^{\mathrm{k}}$; if small to a certain degree, the error between the streamline integral $\mathrm{h}(\mathrm{x})$ and the exact feature $\mathrm{E}(\mathrm{x})$ of the edge will be controlled very small.

It is precisely because of the existence of the flow integral that the basis function of the Bandelet transform can be constructed according to the one-dimensional wavelet function $\theta(t)$. In order to simplify the expression of the basis function of Bandelet transform, we will substitute $\theta_{j, m}(x) \theta_{j, m}(y-h(x))$ the sparse of the Bandelet transform $\langle C m\rangle$ basis function for the geometric image $M$ The expression is:

$$
g_{N}=\sum_{\left|g, C_{m}\right|>T}\left\langle f, C_{m}\right\rangle C_{m}
$$

In addition to obtaining the deterministic error formula of the Bandelet transform approximation, we can get a conclusion. For the Bandelet transform of the $\beta$-order horizon model, the best approximation characteristic can be achieved in a Bandelet band.

\section{Application of Bandelet Transform in Static Human Detection}

Traditional image edge-based representation only describes the image's geometry through edge, not only is not strict, but also it is difficult to describe the image well. To this end, Pennec and Mallat introduced a geometric flow to describe the geometric characteristics of the image, and thus proposed the second generation Bandelet transform. In this paper, a new method of human detection feature extraction based on the second generation Bandelet transform is proposed. The method uses the Bandelet coefficients in Bandelet transform and its statistical characteristics as the features of images, and combines the Linear-SVM classifier to classify and detect the human body in the image.

Any proposed feature extraction method needs to verify its ability to perform human detection in the real world by using the appropriate data set. You must choose a representative data set, not just a certain aspect, or will be detected in the follow-up problems.

For the training and testing of the sample images, the use of a challenging human body database 
-INRIA static human body database. The variation of human posture is relatively large, and the negative sample image has a high similarity with the human body. Dalal and Triggs, as well as many of the current use of this database for human detection research. We extracted 2416 human sample images and 1877 non-human sample images as training set, 1132 human sample images and 821 non-human sample images as test set, the sample size is $128 \times 64$ pixels. For the test of the static body image, the use of the MIT human database, contains 460 still images. Experiments were carried out to select 300 human body images which were meaningful for the human body detection of still images. Each image is an upright human with at least one complete front or back, both horizontal and vertical, at about 500 pixels.

Bandelet transform is used in image compression to minimize the number of non-zero coefficients for the purpose of Bandelet transform parameters will be different, need to be determined through the parameter selection experiments. In the experiment, the same method is used to determine these parameters by comparing the detection ROC curve of the training classifier with different transform parameters. Here, the ROC curve for each possible false positive rate (False Positives Rates) is selected for each detection rate. The higher the curve is, the better the corresponding parameters are.

It can be seen that only one layer of 2-D wavelet transform is used. This is because the higher the number of decomposition layers, the lower the frequency of the loss of the approximation coefficient of the characteristics of characterization capability, greater than the obtained high-frequency detail coefficient of characterization capabilities. Using only one layer of two-dimensional wavelet transform is also conducive to the final detection to extract the detection image of all the scanning window eigenvalues.

Bandelet transform, in theory, j_min smaller, j_max bigger the better, so the establishment of the quadtree is the most reasonable. But the smaller the j_min and the larger the j_max will have the greater the time complexity. If j_min and j_max closer to, even the same and are still very small when the feature is still a good expression, it will greatly reduce the time required for feature extraction. To this end, the following experiment, which level $=1, \mathrm{~T}=15$.

It can be seen that only the $4 \times 4$ block is used to extract the features of Bandelet transform, the characterization ability is the best, and the time consumption is relatively low.

\section{Conclusion}

Based on the basic flow integral of the Bandelet transform, the standard orthogonal basis of the Bandelet transform is constructed based on the theory of the binary wavelet transform and the Bandelet transform approximation is proposed. The basic principle of the Bandelet transform and the approximation properties are studied. Based on the geometric flow and the horizon model, the Bandelet optimal framework of image edge approximation by this property is analyzed. Finally, the application of Bandelet transform in static human detection is studied.

\section{Acknowledgements}

Project Title: Research Projects Supported by the Scientific Research Fund of Yunnan Department of Education

Project Number: 2014Y499

\section{References}

[1] Yang Xiaohui, Wei Li, Jiao Licheng. Image Denoising Based on Second Generation Bandelets and Multi-level Thresholding. Proeeedings of the 6th World Congress on Intelligent Control and Automation, 2006

[2] Dragotti P L, Vetterli M. Shift-Invariant gibbs free denoising algorithm based on wavelet transfoem foot-prints. Proc.SPIE2000, Wavelet Application in Signal and Image Processing, 2000 
[3] Wakin MB, Romberg JK, Choi $\mathrm{H}$ and et al. Rate-distortion optimized image compression using wedgelets. Proc.IEEE Int. Conf, 2001

[4] Do M N. Directiontional multiresolution image representations, 2001

[5] Starck J L, Candes E J, Donoho D L. The curvelet transform for image denoising. IEEE Transactions on Image Processing, 2002 